\title{
Cognitive Interaction Technology
}

\author{
Goals and Perspectives of Excellence Cluster CITEC
}

\author{
Helge Ritter
}

Published online: 21 August 2010

(C) The Author(s) 2010. This article is published with open access at Springerlink.com

\begin{abstract}
The coming decade will bring raw processing power and storage capacities of everyday computers to the same level as small brains. Considering this, current interaction technology seems archaic as it still forces humans to follow highly stereotyped, narrow and often error-prone procedures in order to make computers, robots, or other machinery obey them. The vision behind the Excellence Cluster Cognitive Interaction Technology (CITEC) is to develop ways of interacting with technical systems that are as natural and smooth as communication between humans.
\end{abstract}

\section{Introduction}

With the advances of processor, materials and memory technology, together with novel algorithms and data repositories of unprecedented size, we are approaching the ability to build systems of a tremendous complexity. A major challenge is to shape the interaction of such systems with their environment so that they not only can cope with rich, natural and unstructured environments, but at the same time can also connect well with our human styles of communication and at a semantic level that can be matched to the needs of technical non-experts and lay people [1].

To fulfill these needs, future technology will need cognitive abilities similar to those exhibited by higher developed brains in animals and man. To create the necessary scientific insights, transfer them into technology suitable for applications, and contribute towards better bridges between the technology and the human side summarizes the primary

H. Ritter $(\bowtie)$

Faculty of Technology/CITEC, Bielefeld University, Bielefeld, Germany

e-mail: helge@techfak.uni-bielefeld.de goals of the Bielefeld Excellence Cluster "Cognitive Interaction Technology" (CITEC) [2, 3].

Being aware that this is not only a technological challenge but rather a task that is inseparable from significantly advancing our understanding of cognition itself, CITEC is focussing the efforts of computer scientists, psychologists, linguists, physicists and biologists on the goal of establishing cognitive interfaces that facilitate the use of complex technical systems by supporting a high level of semantic interaction [4]. This combines interdisciplinary, basic, and applied research beyond the classical confines of artificial intelligence. It aims at a thorough understanding of the processes and functional constituents of cognitive interaction in order to replicate it in technical systems at various levels of cognitive complexity ranging from specialized small-scale systems, for example for hand-held devices to display-bound simulated agents in virtual worlds and finally to fully-blown anthropomorphic robots that can aid humans in everyday home settings.

With its focus on interaction and interaction understanding, CITEC complements the Munich Excellence Cluster "Cognition for Technical Systems", which is working towards systems that can act autonomously and at a high level of understanding of their environment. Together, the two initiatives bring together a substantial critical mass of scientists and can create major synergies to tackle the vast field between cognition and technology in pursuit of the vision of a cognitive technology for the future.

\section{Research Profile}

CITEC is structured into four major research areas:

\subsection{Motion Intelligence}

While there are amazing solutions for highly specialized action generation in narrow domains, the synthesis of simi- 

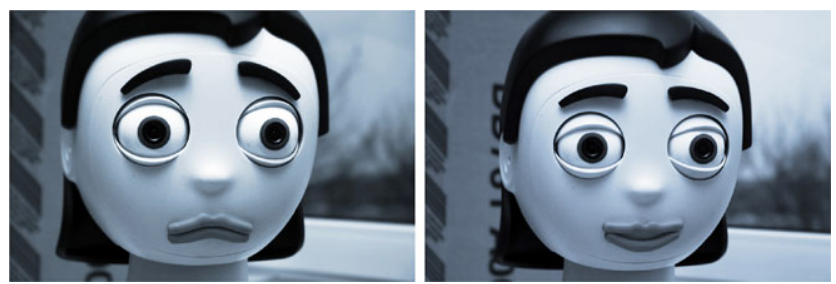

Fig. 1 CITEC emotional humanoid robot head

lar capabilities for unprepared, everyday environments is a major unsolved challenge. Here, major research foci are to consider the control of highly redundant, anthropomorphic actuator systems, such as full-body movements and multifingered hands. Biology, psychology, sports science and robotics groups interact closely to combine algorithmic approaches with insights gained from the analysis of human and animal motion and to come closer to a coherent picture about the internal representation of our movement abilities. A connected research objective is to synthesize robot motions that match better to the expectations of humans in order to achieve smooth cooperation.

\subsection{Attentive Systems}

Research in this area is directed at mechanisms that enable a system to understand and actively focus on what is important, to ignore irrelevant detail, and to share attention with humans, including the important ability of "being social" by providing intuitive, "emotional" feedback (Fig. 1) in order to make robots gain better acceptance and to be understood in a more natural and direct manner. Major goals are to create novel perspectives on attention mechanisms that integrate computational, psychological, and social dimensions, and to create new experimental methodologies for exploring and testing hypotheses about attention mechanisms. To this end, the participating research groups explore the implementation of attention mechanisms at different levels and on a wide spectrum of technical platforms ranging from displaybound attentive user interfaces over augmented reality systems to virtual agents or embodied anthropomorphic robot systems.

\subsection{Situated Communication}

This is a type of communication where the use of language is heavily drawing on the simultaneous presence of shared information in non-linguistic modalities, most notably vision. To elucidate the factors that give situated communication its superb economy and flexibility and to create the necessary integrated perspective for a deeper understanding, linguists and computer scientists are closely collaborating, combining corpora-based research, modeling, theory building, and

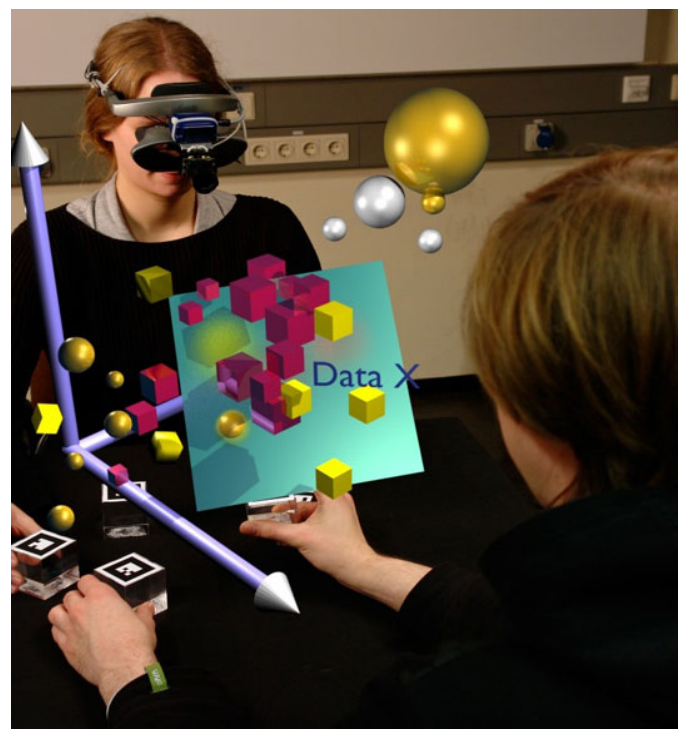

Fig. 2 Exploring novel, AR-based interaction paradigms

the exploration of technical systems. From a technical perspective, an important line of research is the exploration of innovative approaches for enhancing natural and artificial styles of communication by combining visualization, sonification, haptic, and augmented reality devices in novel ways. Fig. 2 depicts a communication situation where configurations of natural objects are linked to data displays made available through AR headsets. Such settings offer novel ways to study how communication is affected by conflicts in situational contexts which may be injected in a highly controlled way through the AR devices.

\subsection{Memory and Learning}

Memory connects perception with experience and allows cognitive processing to generate intelligent action. A major goal is to develop memory systems that can approximate some of the key features of human memory, such as flexible association, scalability and learning at different levels. This raises questions such as: what are the required representations and how do they have to interact with each other? How can we integrate new pieces of knowledge, and what might be the use of forgetting in a technical system?

To address these issues, neuropsychologists and biologists groups working on the brain and its architecture share their insights with sports scientists and robotics researchers. They combine neuropsychological research on human memory with methods for analyzing memory structures from interviews and behavioral studies. This work is complemented by computational approaches exploring biologically inspired memory architectures and combining techniques from the field of datamining with learning concepts ranging from statistical machine learning, neural networks, the 
extraction of ontologies with symbolic methods up to biologically inspired approaches of imitation learning. Another important aspect are developmental studies, analyzing the development of infant cognition as well as communicative principles of parent-infant interaction during the learningby-demonstration process in order to gain novel insights for technical systems, and the interconnection of different modalities to maintain the linkage between conceptual and perceptual levels to enable coherent behavior.

\section{Organization and People}

\subsection{Central Institute}

The EC is constituted as a Central Scientific Institute of the University, allowing it to operate across the borders of the five cooperating faculties (Faculty of Technology (TEC), Faculty of Biology (BIO), Faculty of Physics (PHY), Faculty of Linguistics and Literary Science (LILI), Faculty of Psychology and Sport Science (PSY)) and the European Center for Mechatronics (ECM - an external partner).

Of the current groups, twelve are belonging to the founding principal investigators

1. Philippe Blanchard (PHY)

2. Ralf Möller (TEC)

3. Holk Cruse (BIO)

4. Gert Rickheit (LILI)

5. Paul Drews (ECM)

6. Helge Ritter (TEC)

7. Martin Egelhaaf (BIO)

8. Gerhard Sagerer (TEC)

9. Peter Ladkin (TEC)

10. Thomas Schack (PSY)

11. Hans Markowitsch (PSY)

12. Ipke Wachsmuth (TEC)

Their groups plus two newly created computer science groups (Computer Graphics/Mario Botsch; and Text Technology/Alexander Mehler) bring in a base personnel of about 100 project scientists. Together with the new groups described below, they are supported by an administrative office with a general manager and adjunct managers responsible for the areas public understanding of science, gender issues and financial administration. The scientific affairs of CITEC are directed by a scientific board meeting regularly to coordinate the cluster.

\subsection{New Groups from CITEC funding}

A substantial part of CITEC funding has been invested in the creation of new groups. Three new Computer Science (TEC) groups on
- Semantic Computing (P. Cimiano)

- Theoretical Informatics for Cognitive Systems (B. Hammer)

- Cognitronics and Sensor Systems (U. Rückert),

along with five newly established young investigator tenuretrack groups

- Ambient Intelligence (T. Hermann, TEC)

- Social Agents (S. Kopp TEC)

- Emergentist Semantics (K. Rohlfing PSY)

- Active Sensing (J. Engelmann BIO)

- Gender and Emotion in Cognitive Interaction (F. Eyssel, PSY)

with a last young investigator group on Neuromorphic Engineering in the process of becoming filled.

Additionally, a considerable number of reappointments have been or are currently closely coordinated with the research objectives of CITEC and are partially supported with CITEC-funding: one biology group (V. Dürr-Biological Cybernetics), two linguistics groups (J.P.de Ruiter-Psycholinguistics, P. Wagner-Phonology) and one psychology group (W. Schneider-Cognitive Psychology). Three further re-appointments (PSY, successor of G. Draisbach, BIO, successor of Y. Winter, and Philosophy, successor of A. Beckermann) are currently negotiated.

\section{Central Lab Facilities}

The Central Lab Facilities are a central ressource of CITEC to support the collaboration of the research groups by providing vital technical support for experiments and platform development. Support is along the areas of

- Toolkit Engineering

- Demonstrator Engineering

- HCI experiments

So far supported developments include a virtual environment for medical diagnosis of cognitive skills, an "emotional" humanoid robot head (Fig. 1), the ToBI RobocupHome platform, the DFG MS Science Ship exponat BIRTHE, and a 3D Interaction stage. Another core task is support for complex and technically demanding experiments, such as shown in Fig. 3.

\section{Cooperating Institutions}

An additional strong pillar is the Institute for Cognition and Robotics (CoR-Lab). It is another Central Scientific Institute within Bielefeld University founded on the basis of a special joint funding between Honda Research Europe and 


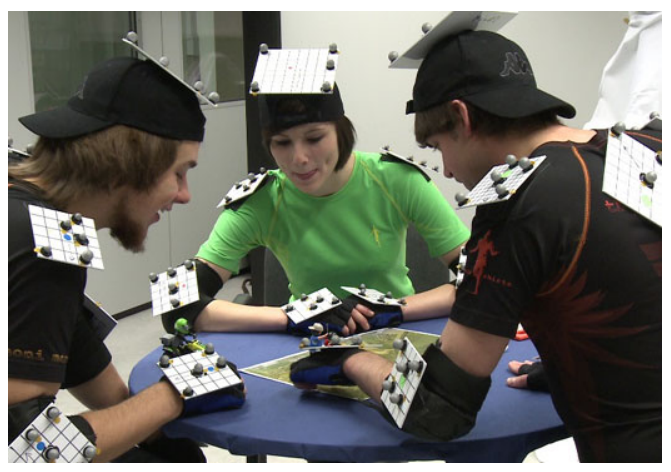

Fig. 3 Combining conversation analysis and motion capture for analysis of social interactions during a discussion

the State of NRW. The researchers in CoR-Lab and its integrated graduate school strengthen CITEC in particular with respect to the sector of cognitive robotics and industrial cooperations in this area, It also offers a unique research laboratory comprising advanced humanoid research robots, including two humanoid ASIMO robots and an iCub system for exploring a wide range of issues of cognitive humanrobot interaction.

This research staff and the additional experience from two Collaborative Research Centers (CRC 360: Situated Artificial Communicators (terminated in 2006) and CRC 673: Alignment in Communication) between the computer science and linguistics groups provides the basis for the above sketched research program.

As a further strengthening measure, CITEC has established a Virtual Faculty consisting of a carefully selected group of renowned scientists from fields pertinent to CIT. These colleagues visit CITEC repeatedly, contributing research seminars and cooperation projects, and in this way add to the profile of CITEC for attracting the best young researchers.

The interaction of the Virtual Faculty with the universitybased researchers is organized through the Center for Interdisciplinary Research (ZiF) - a center for Advanced Study closely operating as a special unit of the University, and the European Center of Mechatronics (ECM) located at the nearby Crassenstein Castle, offering a unique atmosphere for research, tutoring, demonstration and transfer and complementing the $\mathrm{ZiF}$ as an international seminar center for scientists from all over the world.

CITEC has strategic partnerships with key industrial partners to deploy Cognitive Interaction Technology in applications. Envisaged application areas range from "unembodied" CIT in media and information systems to CIT to en- hance functions in typical consumer household applications and finally to the ambitious goal of contributing core technology for shaping the future generation of fully embodied anthropomorphic robot assistants.

Three major partnerships are currently already in place (Bertelsmann AG, Miele Cie. KG; and Honda Research Europe). As an additional partner, WEGE GmbH Bielefeld supports networking activities into local industry.

\section{Promotion of Young Researchers and Gender Issues}

CITEC implements an International Graduate Program comprising distinctive elements such as an embedded $\mathrm{Ca}$ reer Skill Programm, regular Research Retreats to enhance mentoring, International Fellowships encouraging participants to spend one semester at a foreign institution as well as frequent Visiting Lectures by renowned visiting scientists to rapidly advance young scientists to the forefront of their research field, make them part of the international research network, develop their work- and interpersonal skills, and make them strive for excellence.

Open Access This article is distributed under the terms of the Creative Commons Attribution Noncommercial License which permits any noncommercial use, distribution, and reproduction in any medium, provided the original author(s) and source are credited.

\section{References}

1. Ritter H, Sagerer G (2009) Excellence cluster "Cognitive Interaction Technology"- cognition as a basis for natural interaction with technical systems. Inf Technol 2:112-118

2. Excellence Cluster Cognitive Interaction Technology (CITEC Homepage). http://www.cit-ec.de

3. Technology that understands (DFG Video Portal). http://www. excellence-initiative.com/bielefeld-cognitive-interactiontechnology

4. Ritter H, Sagerer G, Dillmann R, Buss M (2009) Human Centered Robot Systems - cognition, interaction, technology. Springer cognitive systems monographs, vol 6 . Springer, Berlin

Helge Ritter joined the Faculty of Technology of Bielefeld University in 1990 after research stays in Finland and USA. He was a Fellow of the Institute for Advanced Studies in Berlin and was awarded the SEL Alcatel Research Prize in 1999 and the Leibniz Prize of the DFG in 2001. $\mathrm{He}$ is a member of the NRW Academy of Sciences, co-founder and Director of the Bielefeld Institute for Cognition and Robotics (CoR$\mathrm{Lab}$ ) and coordinator of the Excellence Cluster Cognitive Interaction Technology (CITEC). 